\title{
Perturbative matching of heavy-light currents at one-loop
}

\author{
Aida X. El-Khadra* and Elvira Gámiz \\ Department of Physics, University of Illinois, Urbana, IL 61801, USA \\ E-mail: 'axk@uiuc edu' megamiz@uiuc edu'
}

\section{Andreas S. Kronfeld}

Theoretical Physics Department, Fermi National Accelerator Laboratory, Batavia, IL 60510,

USA

E-mail: 'askefnal.govi

\section{Matthew A. Nobes}

Department of Physics, Cornell University, Ithaca, NY 14853, USA

and

Brevan Howard Asset Management, London, SW1Y 6XA, United Kingdom

E-mail: 'drnobes @gmaì com,

We present results of a perturbative matching calculation performed at one-loop for heavy-light currents. We use the Fermilab action for the heavy quarks, the Asqtad action for the light quarks, and an improved gluon action. We also present results for heavy-heavy currents with Fermilab heavy quarks and improved glue.

The XXV International Symposium on Lattice Field Theory

July 30-4 August 2007

Regensburg, Germany

\footnotetext{
* Speaker.
} 


\section{Introduction}

The Fermilab Lattice and MILC collaboration's program includes calculations of the hadronic matrix elements for weak $D$ and $B$ meson decays, in particular, the decay constants $f_{D}, f_{D_{s}}, f_{B}$, and $f_{B_{s}}$ and the semileptonic form factors for $B \rightarrow \pi \ell v, D \rightarrow \pi(K) \ell v$, and $B \rightarrow D^{*} \ell v$. In this work we present a perturbative matching calculation of the relevant current renormalizations to one-loop order. The numerical simulations for the above physics analyses use MILC ensembles with improved glue and $2+1$ Asqtad staggered sea quarks [i] $[1]$. The light valence quarks are also generated from Asqtad staggered quarks and converted to naive quarks. The heavy (charm and beauty) quarks are treated with the Fermilab action. See Ref. [2] for more details on the actions and parameters used in the numerical simulations.

\section{Definitions}

In this work we follow the analysis of Ref. [3]-1], where the one-loop corrections to heavy-light and heavy-heavy current renormalizations were calculated for Fermilab heavy and Clover light quarks with Wilson glue.

The heavy-light currents have the form

$$
J_{\mu}^{h l \text { lat }}=\bar{\psi}_{h} \Gamma_{\mu} \psi_{l},
$$

where $\Gamma_{\mu}=\gamma_{\mu}$ or $\gamma_{\mu} \gamma_{5}$ and $\psi_{l}$ denotes a naive Asqtad Dirac spinor. The Fermilab Dirac spinor, $\psi_{h}$, is rotated by

$$
\psi_{h}=\psi\left[1+a d_{1} \gamma \cdot \mathbf{D}\right]
$$

with the tree-level coefficient

$$
d_{1}=\frac{1}{2+m_{0} a}-\frac{1}{2\left(1+m_{0} a\right)} .
$$

The heavy-heavy currents have the form

$$
J_{\mu}^{h h^{\prime} \text { lat }}=\bar{\psi}_{h} \Gamma_{\mu} \psi_{h^{\prime}},
$$

where now both spinors are rotated Dirac spinors. Since the heavy quarks are rotated, the lattice

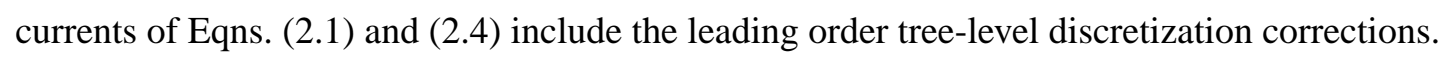

The current renormalization is defined as

$$
Z_{J_{\Gamma}}^{h l}=\frac{\left(Z_{2 h}^{(1 / 2)} \Lambda_{J_{\Gamma}} Z_{2 l}^{(1 / 2)}\right)^{\mathrm{cont}}}{\left(Z_{2 h}^{(1 / 2)} \Lambda_{J_{\Gamma}} Z_{2 l}^{(1 / 2)}\right)^{\text {lat }}},
$$

where $\Lambda_{J_{\Gamma}}$ are the vertex corrections and $Z_{2 h}\left(Z_{2 l}\right)$ are the heavy (light) quark wave function renormalizations.

We factor out the dominant mass dependence due to the tree-level wave function renormalization of the heavy Fermilab quark by defining the perturbative expansion as

$$
e^{-m_{1}^{[0]} a / 2} Z_{J_{\Gamma}}^{h l}=1+g_{0}^{2} Z_{J_{\Gamma}}^{h l[1]}+\ldots,
$$


where the heavy quark masses are defined as usual,

$$
m_{1}^{[0]} a=\log \left(1+m_{0} a\right), \quad m_{0} a=1 /\left(2 \kappa_{h}\right)-1 /\left(2 \kappa_{\text {crit }}\right) .
$$

Since $Z_{V_{4}}$ for degenerate masses is easy to calculate nonperturbatively, it is useful to define

$$
\rho_{J_{\Gamma}}^{h l} \equiv \frac{Z_{J_{\Gamma}}^{h l}}{\sqrt{Z_{V_{4}}^{h h} Z_{V_{4}}^{l l}}}=1+g_{0}^{2} \rho_{J_{\Gamma}}^{[1]}+\ldots
$$

In this case, the dominant mass dependence cancels by construction.

Analogously, for heavy-heavy currents we have:

$$
Z_{J_{\Gamma}}^{h h^{\prime}}=\frac{\left(Z_{2 h}^{(1 / 2)} \Lambda_{J_{\Gamma}} Z_{2 h^{\prime}}^{(1 / 2)}\right)^{\text {cont }}}{\left(Z_{2 h}^{(1 / 2)} \Lambda_{J_{\Gamma}} Z_{2 h^{\prime}}^{(1 / 2)}\right)^{\text {lat }}} .
$$

Taking the leading mass dependence out again, the perturbative expansion is defined as

$$
e^{-\left(m_{1 h}^{[0]}+m_{1 h^{\prime}}^{[0]}\right) a / 2} Z_{J_{\Gamma}}^{h h^{\prime}}=1+g_{0}^{2} Z_{J_{\Gamma}}^{h h^{\prime}[1]}+\ldots
$$

Finally, the $\rho$ factors for heavy-heavy currents are defined as

$$
\rho_{J_{\Gamma}}^{h h^{\prime}} \equiv \frac{Z_{J_{\Gamma}}^{h h^{\prime}}}{\sqrt{Z_{V_{4}}^{h h} Z_{V_{4}}^{h^{\prime} h^{\prime}}}}=1+g_{0}^{2} \rho_{J_{\Gamma}}^{h h^{\prime}[1]}+\ldots
$$

\section{Procedure}

In this work we use the automated perturbation theory techniques developed by Lüscher and Weisz [iיj] to generate the Feynman rules for the lattice actions. We then integrate the loop diagrams by "brute-force" using VEGAS [్ㅗ]. The advantage of using automated perturbation theory is that it is relatively easy to switch actions [6]']. Indeed, we have results for the current renormalizations for two gluon actions, two light quark actions and the heavy quark action.

The one-loop diagrams for the vertex corrections (including the rotations) are given in Ref. [3-1; We have performed the following tests of our calculation:

- For the automated perturbation theory code, we have compared our vertices and propagators against known results.

- We have written two independent programs for calculating the current renormalizations based on the automated perturbation theory code.

- We have a third independent calculation of the current renormalizations using traditional semi-analytic methods.

- Our results for the heavy-heavy currents agree with those of Ref. [iㅣㄱ.] when we switch from the improved gluon propagator to the Wilson gluon propagator. We also reproduce the results of Ref. [్ㅣㅁ] for heavy-light currents with Clover light quarks and Wilson glue.

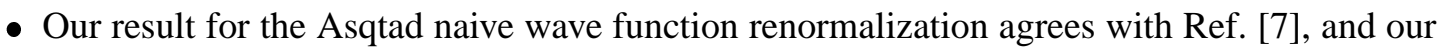
result for the naive-naive vertex correction with Wilson glue agrees with Ref. [8]-1]. 


\section{Results}

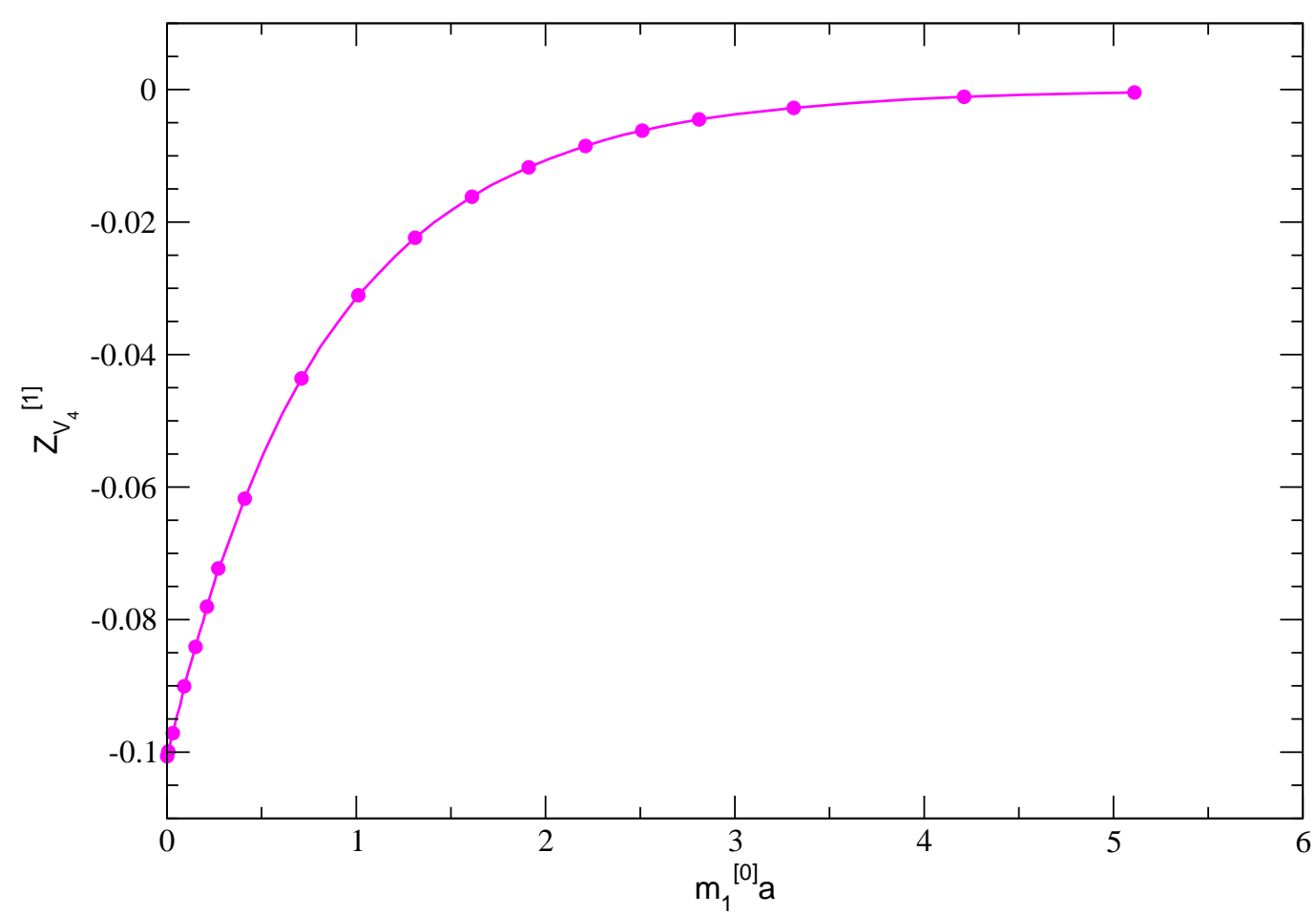

Figure 1: $Z_{V_{4}}^{h h[1]}$ for equal masses as a function of $m_{1}^{[0]}$.

Figures 1-4 illustrate our results for the Z's, $\rho$ 's, and $q^{*}$ 's as functions of the heavy-quark mass, $m_{1}^{[0]} a$. The $q^{*}$ 's are calculated from the log moments using Eqn. (13) of Ref. [9,9].

Our results for the heavy-heavy currents are very similar to those of Ref. [్ㅗ], since they differ only in the gluon propagator. The main features of the mass dependence are the same. Figures 1-2 show results for the degenerate mass $V_{4}$ current. We also have results for the other currents $\left(V_{i}, A_{4}\right.$, $\left.A_{i}\right)$ as well as results for currents with unequal masses. In the massless limit we find

$$
Z_{V_{4}}^{h h[1]}\left(m_{1}^{[0]}=0\right)=-0.10056(3),
$$

in good agreement with Ref. [i]

Figure 3 shows a comparison of the current renormalization of the heavy-naive $A_{4}$ current with the corresponding $\rho$ factor, and Figure 4 shows $\rho_{V_{4}}^{h l[1]}$ and $\rho_{V_{i}}^{h l[1]}$. First, the general features of the

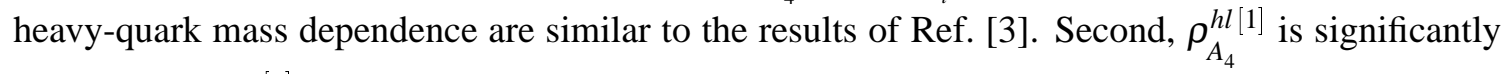
smaller than $Z_{A_{4}}^{h l[1]}$ over the relevant mass range. Hence, the cancellation between the numerator

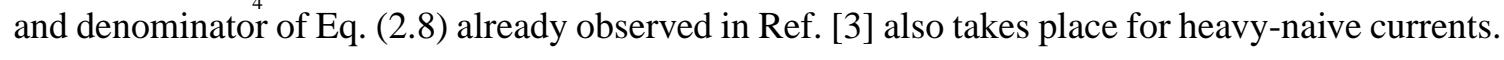




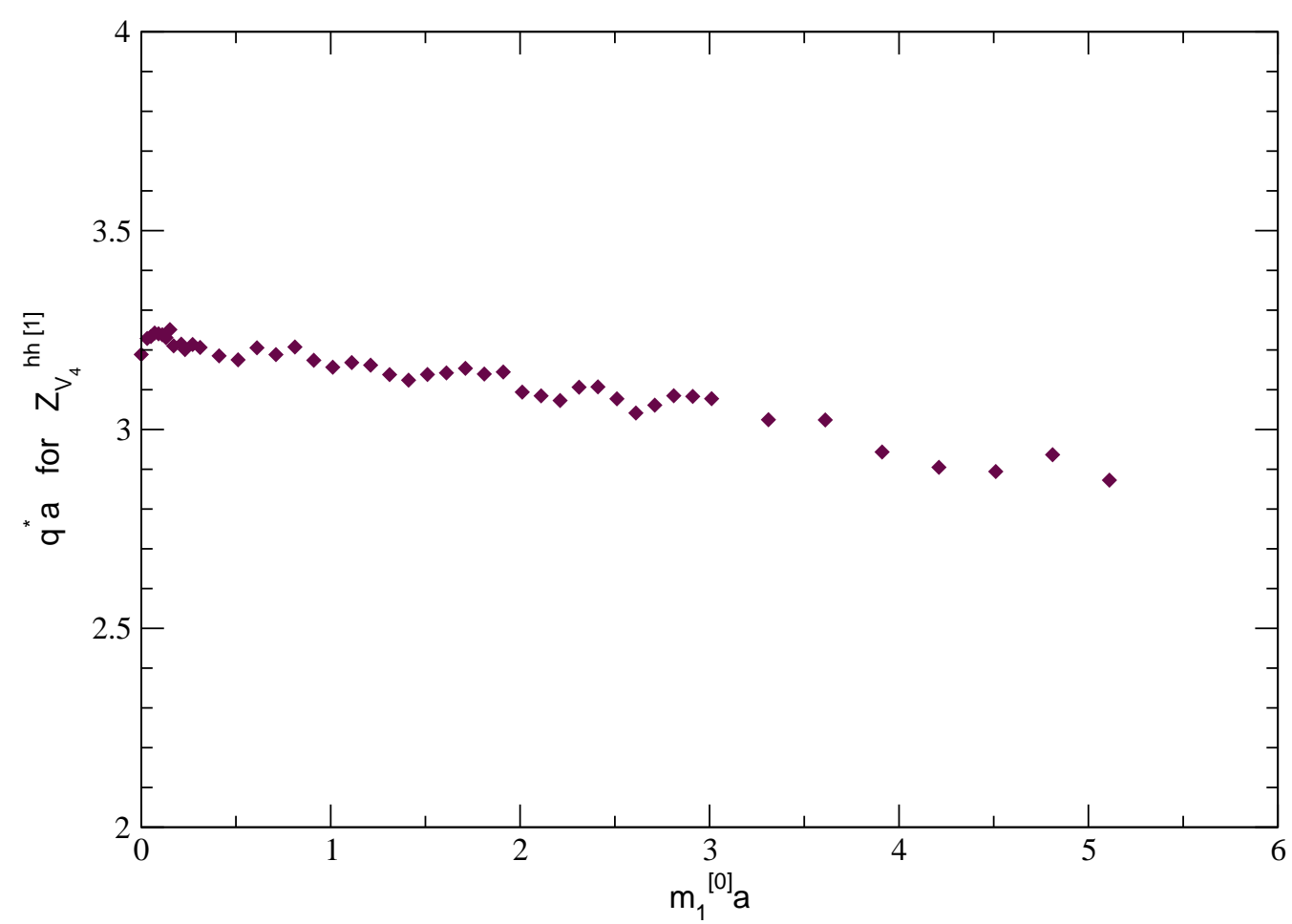

Figure 2: $q^{*} a$ for $Z_{V_{4}}^{h h[1]}$ for equal masses as a function of $m_{1}^{[0]}$.

In the massless limit we find:

$$
\begin{aligned}
& \rho_{V_{4}}^{h l[1]}\left(m_{1}^{[0]}=0\right)=-3.038(2) \cdot 10^{-3} \\
& \rho_{V_{i}}^{h l[1]}\left(m_{1}^{[0]}=0\right)=-3.05(5) \cdot 10^{-3}
\end{aligned}
$$

We also have results for the naive $V_{4}$ current renormalization. In the massless limit we find

$$
Z_{V_{4}}^{l l[1]}\left(m_{0}=0\right)=-0.10457(4)
$$

We have studied the mass dependence of $Z_{V_{4}}^{l l[1]}$ by varying $m_{0}$ between zero and the strange quark mass. We find that $Z_{V_{4}}^{l l[1]}$ is essentially independent of $m_{0}$.

In summary, we have calculated the current renormalizations relevant for the numerical analyses of heavy-light decay constants and semileptonic form factors performed by the Fermilab Lattice and MILC collaborations. We calculate the full mass dependence of the $Z$ 's and $\rho$ 's. The one-loop corrections to the $\rho$ factors are small. They vary roughly between $0.4 \%$ and $4 \%$, depending on lattice spacing. 


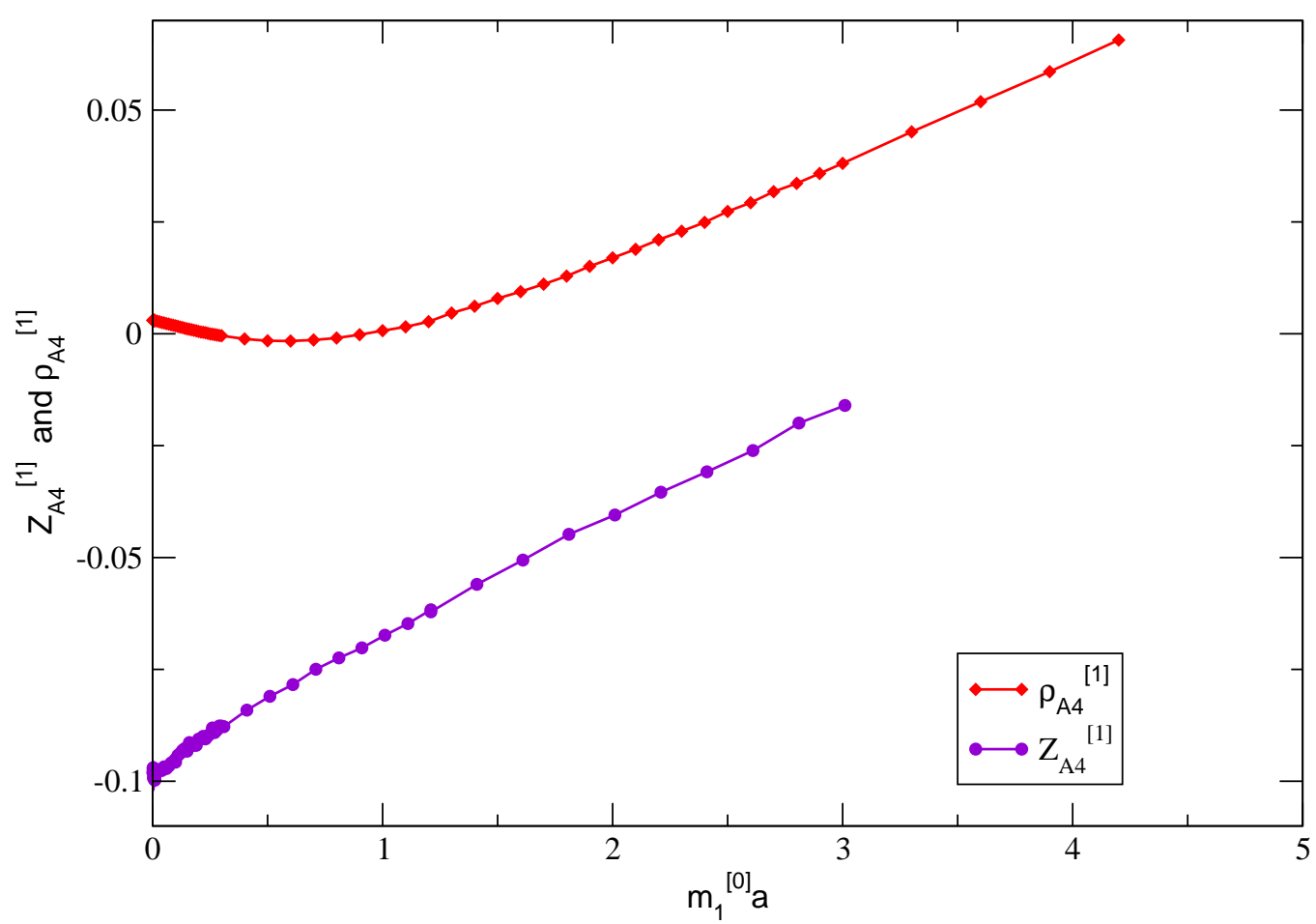

Figure 3: Comparison of $Z_{A_{4}}^{h l[1]}$ with $\rho_{A_{4}}^{h l}$

\section{Acknowledgements}

We thank Howard Trottier for his help with testing M.N.'s automated perturbation theory code, in particular by checking our Asqtad vertices and propagators. This work was supported in part by the DOE under grant no. DE-FG02-91ER40677 and by the Junta de Andalucía [P05-FQM-437 and P06-TIC-02302]. The numerical calculations for this work were carried out on the Fermilab lattice QCD clusters, which are a computing resource of the USQCD collaboration and are funded by the DOE. We are grateful to the Fermilab Computing Divisions for operating and maintaining the clusters. Fermilab is operated by Fermi Research Alliance, LLC, under Contract No. DE-AC0207CH11359 with the United States Department of Energy.

\section{References}

[1] C. Aubin et al., Phys. Rev. D70, 114501 (2004); ibid. D70, 094505 (2004); C. Bernard et al., ibid. D64, 054506 (2001).

[2] C. Aubin et al., Phys. Rev. Lett. 95, 122002 (2005) [hep-lat/0506030]; ibid. 94, 011601 (2005) [hep-ph/0408306]; J. Laiho, these proceedings; P. Mackenzie, these proceedings; J. Simone, these proceedings. 


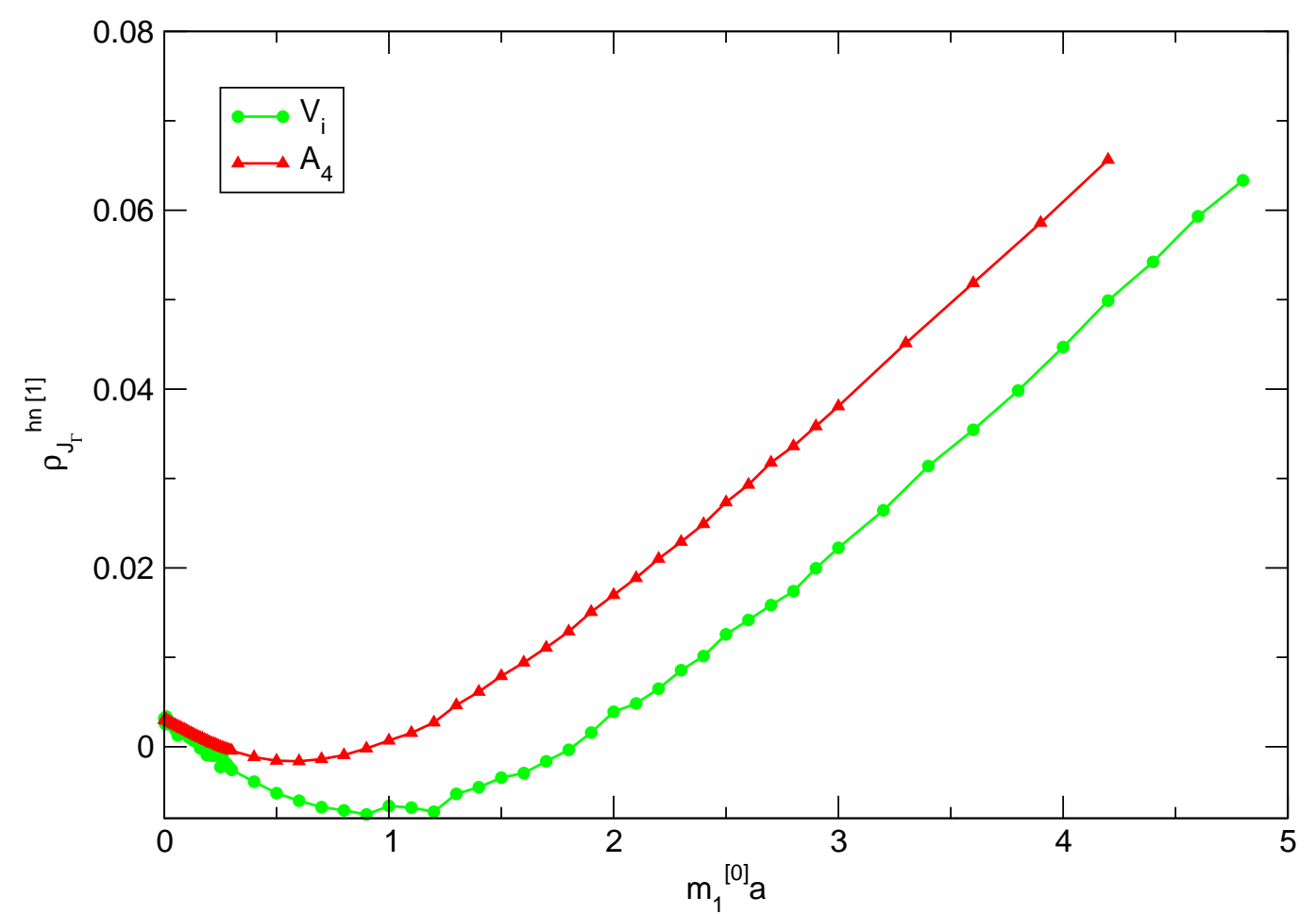

Figure 4: $\rho$ factors for heavy-naive currents

[3] J. Harada, S. Hashimoto, A. S. Kronfeld and T. Onogi, Phys. Rev. D65, 094514 (2002)

[hep-lat/0112045]; J. Harada, S. Hashimoto, K. I. Ishikawa, A. S. Kronfeld, T. Onogi and N. Yamada, ibid. D65, 094513 (2002), [Erratum-ibid. D71, 019903 (2005)] [hep-lat/0112044].

[4] M. Lüscher and P. Weisz, Nucl. Phys. B266, 309 (1986).

[5] G. P. Lepage, J. Comp. Phys. 27, 192 (1978).

[6] H. D. Trottier, Nucl. Phys. Proc. Suppl. 129, 142 (2004) [hep-lat/0310044]; M. A. Nobes and H. D. Trottier, Nucl. Phys. Proc. Suppl. 129, 355 (2004) [hep-lat/0309086].

[7] E. Gulez, J. Shigemitsu and M. Wingate, Phys. Rev. D69, 074501 (2004) [hep-lat/0312017].

[8] W. Lee and S.R. Sharpe, Phys. Rev. D66, 114501 (2002).

[9] K. Hornbostel, G. P. Lepage and C. Morningstar, Phys. Rev. D67, 034023 (2003) [hep-ph/0208224].

[10] Y. Taniguchi and A. Ukawa, Phys. Rev. D58, 114503 (1998) [hep-lat/9806015]; S. Aoki, K. I. Nagai, Y. Taniguchi and A. Ukawa, ibid. D58, 074505 (1998) [hep-lat/9802034]. 